\title{
Características anatómicas y fisiológicas que influyen en la presión intracraneal y la presión de perfusión cerebral en el paciente pediátrico con traumatismo craneoencefálico grave
}

\author{
Anatomical and physiological characteristics that influence intracranial pressure \\ and cerebral perfusion pressure in the pediatric patient with severe head injury.
}

\author{
Daisy Abreu Pérez ${ }^{1}$, Angel Jesús Lacerda Gallardo@1
}

${ }^{1}$ Hospital General Docente "Roberto Rodríguez", Morón, Ciego de Ávila, Cuba.

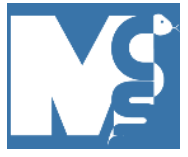

Recibido: 03/10/2020

Revisado: 05/11/2020

Aceptado:05/11/2020

\section{Autor correspondiente}

Daisy Abreu Pérez Hospital General Docente

"Roberto Rodríguez", Cuba

dabreup2016@gmail.com

\section{Conflictos de interés}

Los autores declaran no poseer conflictos de interés.

\section{Fuente de financiación}

Los autores no recibieron apoyo financiero para la investigación, autoría y/o publicación de este artículo.

Este artículo es publicado bajo una licencia de Creative Commons Reconocimiento 4.0 Internacional.

\section{RESUMEN}

La lesión cerebral traumática, es la de mayor potencial para el desarrollo de secuelas devastadoras de todos los tipos de trauma en los niños. La hipertensión intracraneal está presente en más del $65 \%$ de los que sufren de un TCE grave y se relaciona con más de la mitad de las muertes por esta causa. No existe consenso sobre los valores normales de PIC y PPC en los niños, se estima que son dependientes de la edad, pero es un tema en controversia en la actualidad. El objetivo es identificar los elementos anatómicos y fisiológicos en el compartimiento craneal y su contenido en los niños que influyen en las posibles variaciones de los valores de presión intracraneal y de la presión de perfusión cerebral en el paciente menor de 18 años con traumatismo craneoencefálico grave. Las características anatómicas y fisiológicas del paciente pediátrico en sus diferentes etapas de desarrollo definen valores de presión intracraneal y de presión de perfusión cerebral diferentes a los valores en el adulto. Se aborda un tema controversial, que permite identificar aspectos anatómicos y fisiológicos importantes que pueden influir sobre el tratamiento del traumatismo craneoencefálico pediátrico.

Palabras clave: presión intracraneana; presión de perfusión cerebral; traumatismo craneoencefálico.

\section{ABSTRACT}

Traumatic brain injury is one with the greatest potential for the development of devastating sequelae of all types of trauma in children. Intracranial hypertension is present in more than $65 \%$ of those who suffer from a severe TBI and is related to more than half of deaths from this cause. There is no consensus on the normal values of ICP and CPP in children, it is estimated that they are dependent on age, but it is currently a controversial issue. The objective was to identify the anatomical and physiological characteristics in the cranial compartment and its content in children that influence the possible variations in intracranial pressure and cerebral perfusion pressure in patients less than 18 years of age with severe head injury. The anatomical and physiological characteristics of the pediatric patient in their different stages of development define values of intracranial pressure and cerebral perfusion pressure different from those in adults. A controversial issue is addressed, which allows identifying important anatomical and physiological aspects that may influence the treatment of pediatric head trauma.

Keywords: intracranial pressure; cerebral perfusion pressure; traumatic brain injury. 


\section{INTRODUCCIÓN}

La lesión cerebral traumática (LCT), es la de mayor potencial para el desarrollo de secuelas devastadoras, de todos los tipos de trauma en los niños, con un estimado conservador de aproximadamente tres millones de afectados cada año en el mundo (1). Los accidentes de tráfico son la causa más frecuente de LCT en todas las edades (2) y se estima que será la séptima causa de muerte para todos los grupos etáreos en el 2030 (3).

El Traumatismo craneoencefálico (TCE) grave representa entre todas las modalidades de TCE en los niños, alrededor del 3-7 \%, de acuerdo con algunos informes epidemiológicos $(4,5)$. La incidencia de muerte es de 2,8-3,75 / 100000 / año en los países desarrollados, cifras que pudieran incrementarse en los países subdesarrollados o en desarrollo $(6,7)$.

La hipertensión intracraneal (HTI) está presente en más del $65 \%$ de los pacientes que sufren de un TCE grave y se relaciona con más de la mitad de las muertes por esta causa (8). El tiempo de exposición del tejido cerebral a cifras de presión intracraneal (PIC) por encima de los valores considerados normales se relaciona directamente con los resultados (9), por lo que el conocimiento del comportamiento de esta variable se antoja indispensable para su adecuado control.

Los avances en la monitorización multimodal (MMM) de diferentes parámetros de la fisiología intracraneal y el tratamiento neurointensivo derivado de estos, han facilitado el entendimiento y la identificación de los blancos terapéuticos óptimos después de ocurrido el evento traumático en el paciente adulto, sin embargo estos beneficios han sido pobremente aplicados en la población pediátrica, tal es así, que las estrategias para los tratamientos médicos y quirúrgicos óptimos aún son ampliamente debatidos en la comunidad científica mundial $(10,11)$.

La mayoría de la literatura internacional sobre el tema coincide en la existencia limitada de datos sobre el tratamiento de los niños con TCE grave (12), además de la tendencia bastante generalizada de transferir los resultados obtenidos en adultos, que es abrumadoramente superior que en los niños lo cual no es correcto. Un niño no puede ser considerado un adulto pequeño, por lo que resulta necesario identificar las diferencias anatómicas y fisiológicas intracraneales entre un niño y un adulto que permitan su tratamiento diferenciado ajustado a las características de cada grupo de edad, lo cual puede garantizar un tratamiento optimo en cada caso.
El objetivo de esta revisión es aportar información necesaria sobre los en los elementos anatómicos y fisiológicos del niño que influyen en los valores normales, umbrales de tratamiento de la PIC y de la presión de perfusión cerebral (PPC). Para lo cual se revisan las bases de datos nacionales e internacionales sobre el tema.

\section{DESARROLLO}

En el año 2003, aparece publicada la primera edición de las Guías de tratamiento del Traumatismo Craneoencefálico en infantes, niños y adolescentes, patrocinada por La Fundación Americana para el Trauma Cerebral (13). Una actualización de estas guías se realizó en el año 2012 (14), y posteriormente en marzo del 2019, en la misma incluyó cambios en el nivel de evidencias y recomendaciones en las áreas de la neuromonitorización avanzada, la neuroimagenología, la terapia hiperosmolar, la analgesia, la sedación y el bloqueo neuromuscular, el drenaje de líquido cefalorraquídeo, la profilaxis de las crisis convulsivas, el control de la temperatura y la nutrición (15).

Sin embargo, las consideraciones expuestas sobre los valores de PIC para iniciar el tratamiento de la HIC y los valores adecuados de la PPC, se corresponden con los resultados obtenidos en estudios de adultos, sin considerar las diferencias significativas en la fisiología intracraneal entre niños de diferentes edades y entre los niños y los adultos, lo que resulta en extremo controversial.

Las últimas recomendaciones pediátricas de La Fundación Americana para el Trauma Cerebral (BTF), establecen que debe considerarse la monitorización de la PIC en lactantes y niños con TCE grave y se recomienda tratar las elevaciones por encima de 20 $\mathrm{mmHg}$, porque valores por encima de este nivel están asociados a un aumento de la mortalidad (15-17), sin embargo, al considerar este parámetro estrechamente relacionado con la edad y al conocer los valores normales de PIC para diferentes grupos de edad, resulta controversial considerar un mismo valor como umbral de tratamiento para todas las edades.

Consideraciones Anatómicas del cráneo y el cerebro en los niños.

Anatómicamente en el cuerpo de un niño la cabeza es grande y pesada, sostenida por un cuello en el que los músculos no se encuentran debidamente desarrollados y las estructuras ligamentarias son débiles, todo lo cual hace a esta estructura muy vulnerable a los traumatismos $(18,19)$. 
En el recién nacido y el lactante, la cabeza es desproporcionadamente grande con respecto al cuerpo y de forma gradual alcanza un radio cabeza: cuerpo, bastante similar al del adulto en unos pocos años. El crecimiento es particularmente rápido en los primeros años de vida. En el momento del nacimiento, el tamaño del cerebro es el $25 \%$ al del adulto, mientras que el del cuerpo es solo el $5 \%$, la mitad del crecimiento post natal del cerebro ocurre en los dos primeros años de vida, el radio entre el tamaño de la cabeza: largo del cuello, con respecto al largo del cuerpo (alrededor del $25 \%$ ), es dos veces mayor que el de los adultos y constituye un fenómeno continuo de desarrollo y crecimiento desde la etapa gestacional.

El desproporcionado peso de la cabeza limita sus movimientos cuando el niño sufre una caída o cuando es golpeada por un objeto en movimiento, haciéndola más vulnerable a los traumatismos (20-22).

La bóveda craneal, presenta algunas diferencias con la del adulto que pueden influir sobre los cambios fisiológicos que se experimentan después de producido un TCE grave. Nos referimos específicamente al comportamiento de las fontanelas y las suturas, las que experimentan cambios en diferentes momentos.

La fontanela posterior se encuentra cerrada generalmente a los dos meses de edad y la anterior se cierra entre los 12 y 18 meses. La presencia de suturas y fontanelas abiertas pueden ejercer una función "tampón" para la HIC, sobre todo si se produce un incremento gradual de los volúmenes intracraneales, pero este mecanismo es limitado, pues en los niños después de un TCE, los volúmenes se incrementan generalmente de forma rápida y la compleance se agota con facilidad (12).

En los recién nacidos y los lactantes la bóveda craneal es fina, lo que unido a las suturas y fontanelas abiertas pueden facilitar, además, cambios morfológicos ante presiones o fuerzas externas sobre el cráneo $(23,24)$, lo que puede repercutir sobre las estructuras intracraneales y con mayor significación sobre el cerebro.

\section{Consideraciones fisiológicas en la edad pediátrica.}

En términos de fisiología y fisiopatología los niños no se comportan de forma similar a los adultos. Resulta un error muy frecuente que los profesionales encargados del tratamiento de niños que sufren de un TCE los consideren como un adulto pequeño y que, en consecuencia, se extrapolen los resultados obtenidos en estudios de adultos a la edad pediátrica.

En el mejor de los casos esta práctica es desacertada y en el peor puede llegar a ser peligrosa. Sucede que la disponibilidad de estudios sobre el tema en niños es mucho menor, por lo que también lo es la evidencia en qué sustentar las recomendaciones.

En la edad pediátrica es controversial establecer valores absolutos para diferentes edades con respecto a la definición de los umbrales para iniciar el tratamiento de la HIC, debido a que las peculiaridades fisiológicas que existen en el desarrollo de la bóveda craneal en los niños lo impide, por tanto, este acápite no queda bien definido en la actualidad por su dependencia y estrecha relación con los grupos de edades.

De igual manera sucede con los valores de la presión arterial sistémica (PAS), que varían con la edad. Existe una estrecha relación entre la PAS, la presión arterial media (PAM) y la PPC, las variaciones de la primera tienen influencia directa sobre los valores de PPC, por lo que estos, también pueden variar en cada grupo de edad, lo que haría muy controversial considerar un umbral de tratamiento absoluto en este aspecto para todos (25).

Existen diferencias considerables entre los niños y los adultos en el ámbito del metabolismo y los índices cambian constantemente durante el reposo y en la actividad física, hasta lograr una estabilidad en la adultez.

Algunos detalles fisiológicos guardan importancia para las especialidades quirúrgicas. Los niños tienen un menor volumen sanguíneo circulante y una menor tolerancia a las pérdidas hemáticas, muestran además un mayor riesgo ante largos períodos de anestesia general, reaccionan de forma diferente a los medicamentos y tienen una reducción en la perfusión tisular, todos estos elementos deben ser considerados en el momento de someter a un niño a un procedimiento neuroquirúrgico en cualesquiera de sus variantes.

Variables intracraneales relacionadas con el TCE.

Presión intracraneal en el niño.

La PIC se define como la presión que existe dentro de la bóveda craneal. Se ha establecido que el funcionamiento cerebral es adecuado con valores de PIC entre 10 y $15 \mathrm{mmHg}$ en adultos, de 3 a $7 \mathrm{mmHg}$ en niños y de 1,5 a $6 \mathrm{mmHg}$ en recién nacidos (26), sin embargo, el umbral para iniciar el tratamiento en pacientes pediátricos con TCE grave al que se asocia una HIC, no se encuentra debidamente establecido.

Aunque puede ser evaluada de diferentes formas, la PIC generalmente es estimada a través de la presión que ejerce el LCR sobre las paredes del sistema 
ventricular, por lo que pudiera ser considerada como la mayor presión ejercida sobre una aguja colocada dentro del sistema ventricular, para evitar la pérdida del fluido (27).

La estática y la dinámica del LCR están fundamentados en cuatro parámetros: 1 . La velocidad de producción, 2. La compleance definida por la relación exponencial entre la presión de LCR y el volumen, 3. La resistencia a la absorción y 4. La presión en el seno venoso intradural (27).

Tras el cierre de las suturas y las fontanelas, el cráneo se convierte en una estructura inextensible $y$, por tanto, mantiene un volumen constante independientemente de su contenido. En condiciones normales, este contenido se puede dividir en tres compartimentos: parénquima cerebral (80\%), líquido cefalorraquídeo (LCR) (10 \%) y sangre (10\%).

Cuando aumenta el volumen de alguno de estos componentes, aumenta también la presión que ejerce dicho compartimento sobre los otros dos y se convierte en un mecanismo "tampón" para la PIC, lo que se conoce como teoría o doctrina de Monroe-Kellie.
En condiciones normales, estas variaciones se compensan de forma aguda a través del desplazamiento del LCR hacia la cisterna terminal. De forma más tardía, existe una disminución del flujo cerebral. Solo en situaciones crónicas, el parénquima es capaz de deformarse, a expensas de perder parte del agua extracelular e incluso neuronas y glía.

Sin embargo, cuando estos mecanismos tampón fallan, el aumento de la PIC puede suponer una disminución en el aporte sanguíneo y secundariamente una reducción de la PPC, con lo que aumenta la probabilidad de lesiones isquémicas, pues la variable depende tanto de la PAM como de la PIC (26).

En esta relación se distinguen tres situaciones: en una primera fase, el aumento del volumen intracraneal (VI) no repercute en la PIC pues el desplazamiento del LCR y del volumen sanguíneo cerebral lo compensa. En la segunda fase, el sistema de regulación se encuentra en el límite y no consigue amortiguar el aumento de presión secundario al aumento de volumen. Por último, en la tercera fase, el sistema de autorregulación ha desaparecido y pequeños cambios de volumen suponen elevaciones muy llamativas de la PIC (27). (Figura 1) y (Figura 2).

\section{FIGURA 1. NEUROMONITORIZACIÓN CONTINUA DE LA PIC EN UN NIÑO CON TCE GRAVE}

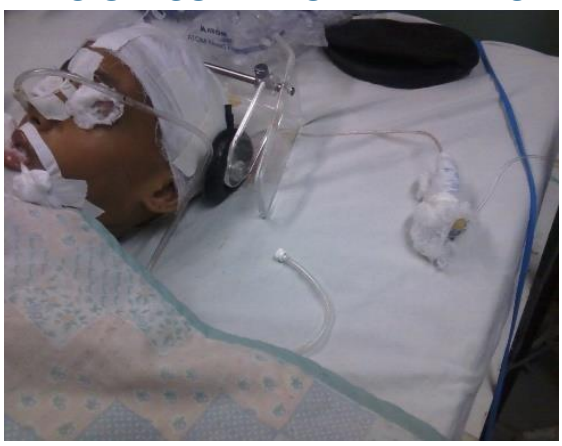

FIGURA 2. REPRESENTACIÓN GRÁFICA DE LA MONITORIZACIÓN CONTINUA DE LA PIC EN UCIP

Análisis espectral del cambio de la morfología cuando la PIC asciende por encima de los $20 \mathrm{mmHg}$. A) PIC normal. B) PIC anormal.

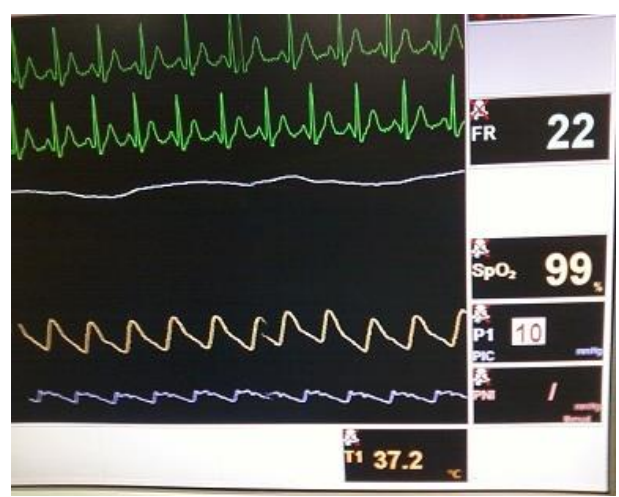

A)

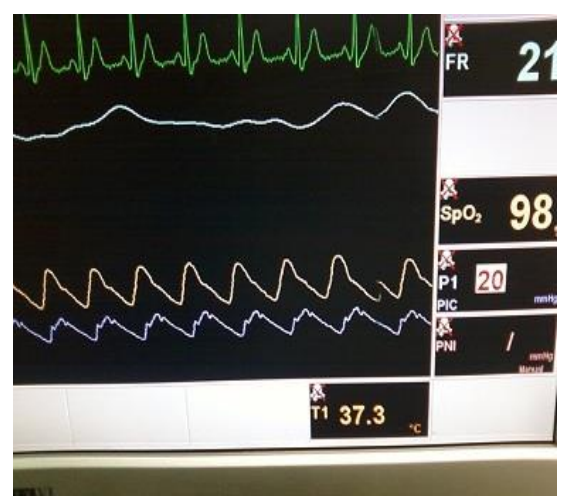

B) 
Consideramos valores normales de PIC en el recién nacido de 0-2mmhg, en el niño de 1 mes a 11 meses y 29 días de 3-6 $\mathrm{mmHg}$, con umbrales de tratamiento por encima de $6 \mathrm{mmhg}$, en el niño mayor de un año de 3$15 \mathrm{mmhg}$, dividiéndolo de 1-4 años por encima de 10 $\mathrm{mmHg}$ y de 5-17 años, 11 meses y 29 días por encima de $15 \mathrm{mmHg}$ (28).

\section{Presión de perfusión cerebral}

La PPC se define como el gradiente de presión a través del lecho vascular cerebral. La PPC resulta de restar la PIC a la presión arterial media (PAM) (29). El proceso de autorregulación cerebral permite la variabilidad de la resistencia cerebrovascular con la consiguiente conservación del FSC ante cambios en la PPC, procesos que se encuentran alterados en el TCE $g$ del niño, acarreando ante disminución de la PPC caída del FSC e isquemia.

Las opiniones sobre la PPC óptima y su relación con la autorregulación cerebral y el pronóstico han evolucionado a lo largo de los años. Según las últimas recomendaciones pediátricas de 2019 de la BTF, la PPC mínima en niños con TCE grave está entre $40-50 \mathrm{mmHg}$ (15). En adultos, el valor de PPC recomendada para la supervivencia y los resultados favorables es entre 60 y $70 \mathrm{mmHg}$ (30).

La edad pediátrica establece diferencias en relación a los pacientes mayores de 18 años, en cuanto a los valores de PAM, del FSC y por la tasa metabólica cerebral de oxigeno (26). A pesar de lo cual no existen suficientes estudios que permitan conocer los valores adecuados en los diferentes grupos de edades pediátricas y su asociación con los resultados.

Los valores de PAM se obtienen por la fórmula $2 \mathrm{x}$ presión diastólica + presión sistólica/3, oscilando entre el 5 y 90 percentil como rango de normalidad, de acuerdo a cada grupo de edad, por debajo del 5 percentil, se considera hipotensión arterial y por encima del 90 hipertensión arterial. Al presentar una PAM en el niño más baja que en el adulto y restarle las cifras de PIC que son menores igualmente que en el adulto $(6,10$ y $15 \mathrm{mmHg}$ por edades), obtendremos como resultado valores de PPC menores que en el paciente mayor de 18 años.

En el lactante, según las curvas de percentiles para la TA oscila de 53,3-82,6 mmHg con una media de 67,9 mmHg. De 1-4 años de 56,6-83,3 mmHg con una media de 69,9 mmHg. De 5-17 años, 11 meses y 29 días de 65$106,6 \mathrm{mmHg}$ media de $85,5 \mathrm{mmHg}$ (31). Utilizamos el valor inferior de la TA (5to percentil) y se le resta el umbral de tratamiento de la PIC en cada grupo etáreo $(6,10,15 \mathrm{mmHg})$ obteniéndose las siguientes PPC: lactantes $53-6=47 \mathrm{mmHg}$, de $1-4$ años $57-10=47$ $\mathrm{mmHg}, 65-15=50 \mathrm{mmHg}$ (28).

Presión de perfusión cerebral optima (PPC Opt.) y el índice de reactividad cerebrovascular (PRX).

El PRx se calcula mediante el coeficiente de correlación de Pearson entre la PIC y la PAM. El PRx puede considerarse un estimador de autorregulación cerebral. Un PRx negativo indica una autorregulación intacta, mientras que un PRx positivo indica autorregulación deteriorada. Mediante la aplicación de un método automatizado de ajuste de curvas se puede determinar la PPC al valor mínimo de PRx y calcular el PPC opt.

En el TCE pediátrico, los pacientes con un menor PRx tienen una mayor probabilidad de supervivencia. Si bien no se han diseñado grandes estudios para determinar la PPC opt. en niños, se ha propuesto una PPC crítica para poblaciones estratificadas de edad en niños. Específicamente, en los grupos de edad 2-6, 710 y 11-16 años de edad, los buenos resultados se asociaron con valores de PPC de 43, 54 y $58 \mathrm{mmHg}$, respectivamente (17).

Utilizando los valores de deferentes PPC se ha observado que cuando los valores se desvían del óptimo hay una relación entre los resultados y la diferencia de presión. En particular, los que pasan más tiempo con PPC menor que PPC opt. muestra un peor resultado (9).

También se ha objetivado una relación significativa entre el pronóstico y la desviación de la PPC opt. Estos hallazgos destacan la importancia de guiar el tratamiento del TCE usando índices de autorregulación9. En la práctica clínica se puede interpretar que debe evitarse las diferencias entre el PPC real y PPC opt, mientras que el objetivo deben ser valores superiores que $10 \mathrm{mmHg}$. Estas observaciones preliminares apoyan la necesidad de investigación sobre la viabilidad de la terapia orientada a la PPC opt.

\section{CONCLUSIÓN}

El establecimiento de valores para el tratamiento de la PIC y el mantenimiento de la PPC en el paciente pediátrico es indispensable, permitiendo influir de forma directa sobre los resultados. Conocer las características anatómicas y fisiológicas de estas etapas de la vida es importante para el personal encargado de tratar a los niños, así como establecer las diferencias en relación al adulto. 


\section{REFERENCIAS}

1. Dewan MC, Mummareddy N, Wellons JC, Bonfield CM. Epidemiology ofGlobal Pediatric Traumatic Brain Injury: Qualitative Review. World Neurosurg 2016; 91:497-509.e1. https://doi.org/10.1016/i.wneu.2016.03.045

2. Hari HD, Siddharth C. Management of traumatic brain injury patients. Korean J Anesthesiol 2018;71(1):12-21. https://dx.doi.org/10.4097\%2Fkjae.2018.71.1.12

3. Hankinson TC, Beauchamp K. Pediatric Traumatic Brain Injury: The Global View. World Neurosurg 2016; 92:540541. https://doi.org/10.1016/j.wneu.2016.06.012

4. Amaranath JE, Ramanan M, Reagh J, Saekang E, Prasad N, Chaseling $R$, et al. Epidemiology of traumatic head injury from a major paediatric trauma centre in New South Wales, Australia. ANZ J Surg 2014; 84:424-428. https://doi.org/10.1111/ans.12445

5. Ferreros I, Peiro S, Chirivella-Garrido J, Duque P, Gagliardo $P$, Perez-Vicente $R$, et al. Incidencia de la hospitalización por traumatismo craneoencefálico en la infancia y adolescencia (Comunidad Valenciana, 2002-2009). Rev Neurol 2012; 54:719-728. https://doi.org/10.33588/rn.5412.2011701

6. Bowman SM, Bird TM, Aitken ME, Tilford JM. Trends in hospitalizations associated with pediatric traumatic brain injuries. Pediatrics 2008; 122:988-993. https://doi.org/10.1542/peds.2007-3511

7. Mitra B, Cameron PA, Butt W, Rosenfeld JV. Children or young adults? A population-based study on adolescent head injury. ANZ J Surg 2006; 76:343-350. https://doi.org/10.1111/j.1445-2197.2006.03723.x

8. Agrawal S, Branco RG. Neuroprotective measures in children with traumatic brain injury. World J Crit Care Med 2016; $5(1): 36-46$. https://dx.doi.org/10.5492\%2Fwjccm.v5.i1.36

9. Young AMH, Donnelly J, Czosnyka M, Jalloh I, Liu X, Aries $\mathrm{MJ}$, et al. Continuous multimodality monitoring in children after traumatic brain injury-preliminary experience. PLoS One 2016; https://doi.org/10.1371/journal.pone.0148817

10. Young $\mathrm{AMH}$, Kolias AG, Hutchinson PJ. Decompressive craniectomy for traumatic intracranial hypertension: application in children. Childs Nerv Syst 2017; 33:17451750. https://doi.org/10.1007/s00381-017-3534-7

11. Figaji AA, Fieggen AG, Peter JC. Early decompressive craniotomy in children with severe traumatic brain injury. Childs Nerv Syst 2003; 19(9):666-673. https://doi.org/10.1007/s00381-003-0804-3

12. Figaji AA. Anatomical and Physiological Differences between Children and Adults Relevant to Traumatic Brain Injury and the Implications for Clinical Assessment and Care. Front Neurol 2017; 8:685. https://doi.org/10.3389/fneur.2017.00685

13. Adelson PD, Bratton SL, Carney NA. Guidelines for the acute medical management of severe traumatic brain injury in infants, children, and adolescents. Pediatr Crit Care Med. 2003; 4:S1-S75. URL.

14. Kochanek PM, Carney N, Adelson PD. Guidelines for the acute medical management of severe traumatic brain injury in infants, children, and adolescents-second edition. Pediatr Crit Care. Med. 2012; 13 (Suppl 1):S1-S82. https://doi.org/10.1097/pcc.0b013e31823f435c

15. Kochanek PM, Tasker RC, Carney NA, Totten AM. Guidelines for the Management of Pediatric Severe Traumatic Brain Injury, Third Edition: Update of the Brain Trauma Foundation Guidelines. Pediatr Crit Care Med 2019; 20:S1-S82. https://doi.org/10.1093/neuros/nyz051
16. Arunkumar S, Devi BI, Shukla D, et al. Is intracranial pressure monitoring useful in children with severe traumatic brain injury? Neurol India 2016; 64: 958-61. https://doi.org/10.4103/0028-3886.190259

17. Rossich Verdés R. Avances en neuromonitorización. Rev Esp Pediatr 2017; 73(Supl. 1): 59-61. URL.

18. Tunthanathip T, Phuenpathom N. Impact of road traffic injury to pediatric traumatic brain injury in Southern Thailand. J Neurosci Rural Pract 2017; 8(4):601-608. https://doi.org/10.4103/jnrp.jnrp 38117

19. Lavinia Zorilă $A$, Valentin Zorilă $M$, Cristian Marinaş $M$, Ştefan Jolescu R, Lucian Zorilă G, Florou C, et al. Evaluation of brain injuries in children deceased due to head trauma. Rom J Morphol Embryol 2017, 58(4):1417-1428. URL.

20. Burdi AR, Huelke DF, Snyder RG, Lowrey GH. Infants and children in the adult world of automobile safety design: pediatric and anatomical considerations for design of child restraints. J Biomech 1969;2(3):267-80. https://doi.org/10.1016/0021-9290(69)90083-9

21. Ludwig S, Loiselle J. Anatomy, growth, and development. In: Eichelberger MR, editor. Pediatric Trauma: Prevention, Acute Care, Rehabilitation. St. Louis, Missouri: Mosby-Year Book, Inc (1993). p. 39-58.

22. Cory CZ, Jones MD, James DS, Leadbeatter S, Nokes LD. The potential and limitations of utilising head impact injury models to assess the likelihood of significant head injury in infants after a fall. Forensic Sci Int 2001; 123(2-3): 89-106. https://doi.org/10.1016/s0379-0738(01)00523-0

23. Prange MT, Luck JF, Dibb A, Van Ee CA, Nightingale RW, Myers BS. Mechanical properties and anthropometry of the human infant head. Stapp Car Crash J 2004; 48:279-299. URL.

24. Margulies SS, Thibault KL. Infant skull and suture properties: measurements and implications for mechanisms of pediatric brain injury. J Biomech Eng 2000; 122(4):364-71. https://doi.org/10.1115/1.1287160

25. Allen BB, Chiu Y, Gerber LM, Ghajar J, Greenfield JP. AgeSpecific Cerebral Perfusion Pressure Thresholds and Survival in Children and Adolescents with Severe Traumatic Brain Injury. Pediatr Crit Care Med. 2014; 15(1): 62-70. https://doi.org/10.1097/pcc.0b013e3182a556ea

26. Rodríguez-Botoa G, Rivero-Garvía M, Gutiérrez-González R, Márquez-Rivas J. Conceptos básicos sobre la fisiopatología cerebral y la monitorización de la presión intracraneal. Neurología 2015; (1):16-22. https://dx.doi.org/10.1016/j.nrl.2012.09.002

27. Shahlaie K, Zwienenberg-Lee M, Muizelaar JP. Clinical Pathophysiology of Traumatic Brain Injury. In: Winn HR, editors. Youmans and Winn Neurological Surgery. Seventh edition. Philadelphia: Elsevier, 2017. p. 2843-2859.

28. Abreu PD, Lacerda GA, Díaz AJ, Martín CD. Neuromonitorización en el trauma craneoencefálico grave en Pediatría. Neurocirugía 2016;27(4):176-185. https://doi.org/10.1016/j.neucir.2015.11.004

29. Steiner LA, Andrews PJ. Monitoring the injured brain: ICP and CBF. Br J Anaesth 2006; 97:26-38. https://doi.org/10.1093/bja/ael110

30. Carney N, Totten AM, O'Reilly C, Ullman JS, Hawryluk GWJ, Bell MJ, et al. Guidelines for the Management of Severe Traumatic Brain Injury, Fourth Edition. Neurosurgery. 2017;80(1):6-15. https://doi.org/10.1227/neu.0000000000001432

31. Arjona VD, LLedín BM. Valores normales en la función hemodinámicas. En: Ruza TF. Manual de Cuidados Intensivos Pediátricos. 2d edición, Madrid, España, Capitel Ediciones, 2010. p. 312-314. 